\title{
Damage Analysis of Tattoo Needle Tips
}

\author{
Lilla ASZTALOS, ${ }^{1}$ Borbála LEVELES ${ }^{2}$ \\ Budapest University of Technology and Economics, Faculty of Mechanical Engineering, Department of \\ Materials Science and Engineering, Budapest, Hungary \\ ${ }^{1}$ lilla@eik.bme.hu \\ ${ }^{2}$ levelesb@gmail.com
}

\begin{abstract}
Tattooing is becoming more and more accepted at different levels of society today. A contributor to this is that besides body decoration, the cosmetics industry is increasingly using it for make-up tattoos and to hide skin imperfections and surgical scars. Tattoo needles, despite being in direct contact with human tissues and even with blood, are not subject to current Medical Device Regulation, so they do not require a number of material and biocompatibility tests in order to be placed on the market. The focus of our research was on how the needle and the soldering of the needles are damaged during tattooing, and how this develops over time, as a worn needle tip can not only degrade the quality of the tattoo, but also increase skin breakdown and the amount of dissolving allergenic substances.
\end{abstract}

Keywords: tattoo needle, damage, needle wear.

\section{Introduction}

Tattoos are created by piercing tiny needles at a frequency of $50-3000 \mathrm{~Hz}$ into the skin. The needles then break through the surface of the skin and introduce the dye into the skin's dermis layer (min. $1.2 \mathrm{~mm}$ deep). The tattoo dye is perceived by the skin as a foreign substance and initiates macrophage cells to destroy the dye, however, after ingestion of the dye, the cells get stuck with the dye in the skin matrix. After 2-3 weeks of creating the tattoo, the colours fade, as the tattoo also gets dye in the upper epidermis, which wears out over time as the cells die and regenerate. Over the years, the tattoo also fades as the dye in the skin is gradually broken down by the immune system [1-3].

\subsection{Needle tip damage}

Needle tip destruction is typically studied in medical applications such as injection needles or thicker needles used in epidural anaesthesia. The tip of the spinal needle is often damaged during the latter, especially when the spinal cord puncture needs to be repeated several times into the bone or cartilage due to failure, so it is of primary interest for us to examine these. [4-6]. Because these are all disposable needles, they remain intact or slightly deformed after a puncture. In clinical practice, some research groups examined Quincke-type needles used for epidural anaesthesia and found that four percent of the needle tips were clearly bent and $11 \%$ were slightly bent based on scanning electron micrographs. Needles in contact with the bone caused clear tip damage in $7 \%$, and $99 \%$ of the needles remained intact or slightly bent in the case of penetration without bone contact. In conclusion, the tips of disposable needles are suffer more damage in contact with hard tissue (bone) than after penetration into soft tissue [4, 7].

Like injection needles, thin tattoo needles suffer small but permanent damage after contact with the skin. The longer a needle used, the more the tip becomes blunted, which causes more pain during use, and bacteria are better able to adhere to surface damage, which also increases the risk of infection [8-10]. The study of this destruction is worth a closer look, as it can also cause unwanted damage to the skin surface, and the tattooing process can be more painful. The dullness of the nee- 


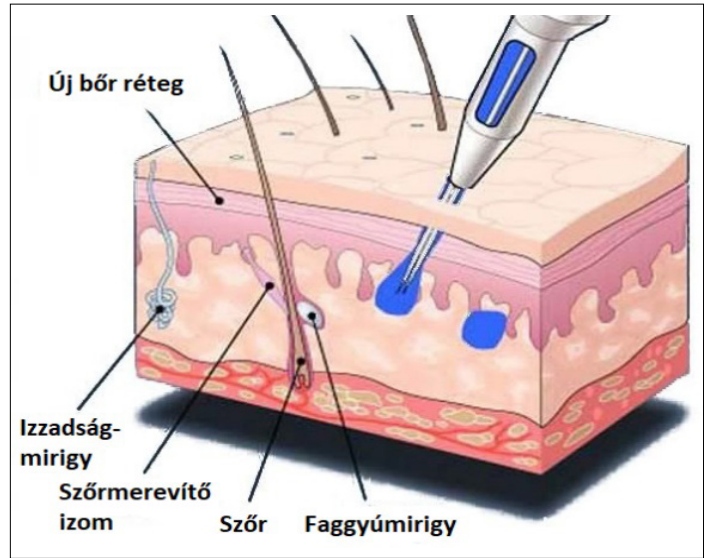

Figure 1. Tattooing process.
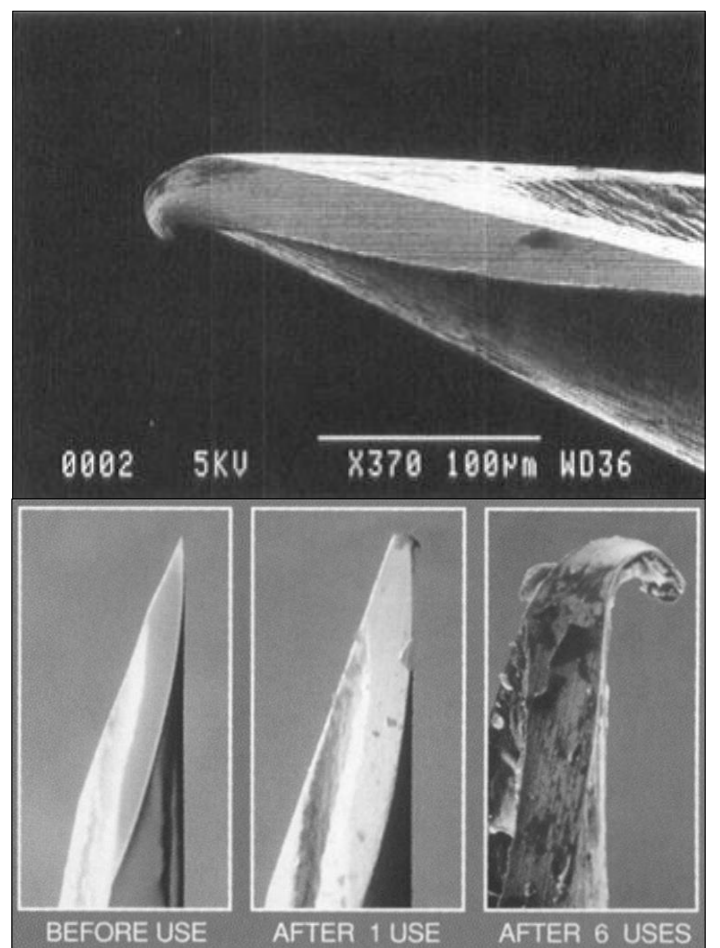

Figure 2. Abrasion of needle tips used for epidural anesthesia [4].

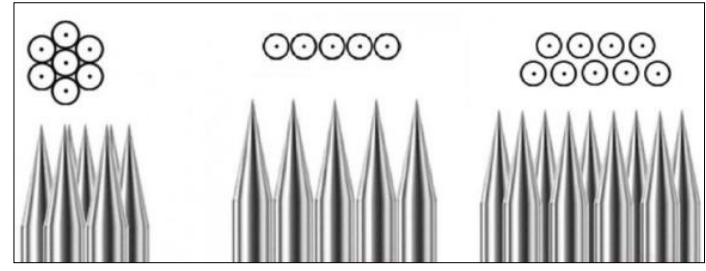

Figure 3. Tattoo needle arrangements. From left to right: round, flat and magnum layout. dle tips also leads to a deterioration in the quality of the tattoo, so it should be replaced consistently, over a period of time or after the tattooed surface. To avoid this, we aimed to examine how long generally - or after a tattoo - it is advisable to replace the needle tip so that maximum utilization is achieved. Of course, at the beginning of each new tattoo, the artist must use a new, sterile needle, for hygiene reasons.

\section{Methods}

\subsection{In vivo damaged needles}

To examine the needle tips, we used 13 round arranged tattoo needles pierced in areas of 30-100 $\mathrm{mm}^{2}$. Each needle was used to make a tattoo using the handpoke (freehand tattoo) method, meaning that the needles were not moved by machine, but were inserted into the skin by hand. After use, the dried paint and impurities were removed with an ultrasonic vibrator, ethyl alcohol, and acetone, but still remained in the wells. In addition, 2 unopened, sterile needles were examined as a reference. These needles are individually soldered in different numbers, there are also 3-5-9 pieced ones in three different arrangements (Figure 3.). After cleaning, the needles were examined by stereo microscopy, and then scanning electron microscopy (SEM) images of the tips and soldering were taken to see how much they had cracked during use.

\subsection{Modeling the tattooing process in an in vitro environment}

As it is difficult to determine the period of use and the compressive force of hand-used needles, it was advisable to devise a standardized measurement method to set the wear time of the needles. For the first experiment, we handmade samples on the so-called training skin used by tattooists, and then in order to make the abrasion process as even as possible per unit of time and for easier feasibility, we mounted a two-roll tattoo device from the brand Horizon to a Yamaha LCM100 linearly controlled robot in the BME ATT laboratory (Figure 4.). The manual tattooing process was performed for $30 \mathrm{~min}$ and the automated process for 30,60 , and $90 \mathrm{~min}$. The power supply was set to a specific frequency, to a value between 50 and $60 \mathrm{~Hz}$ determined from highspeed camera recording. This represents about a hundred thousand insertions of with a needle that has been running for 30 minutes. 


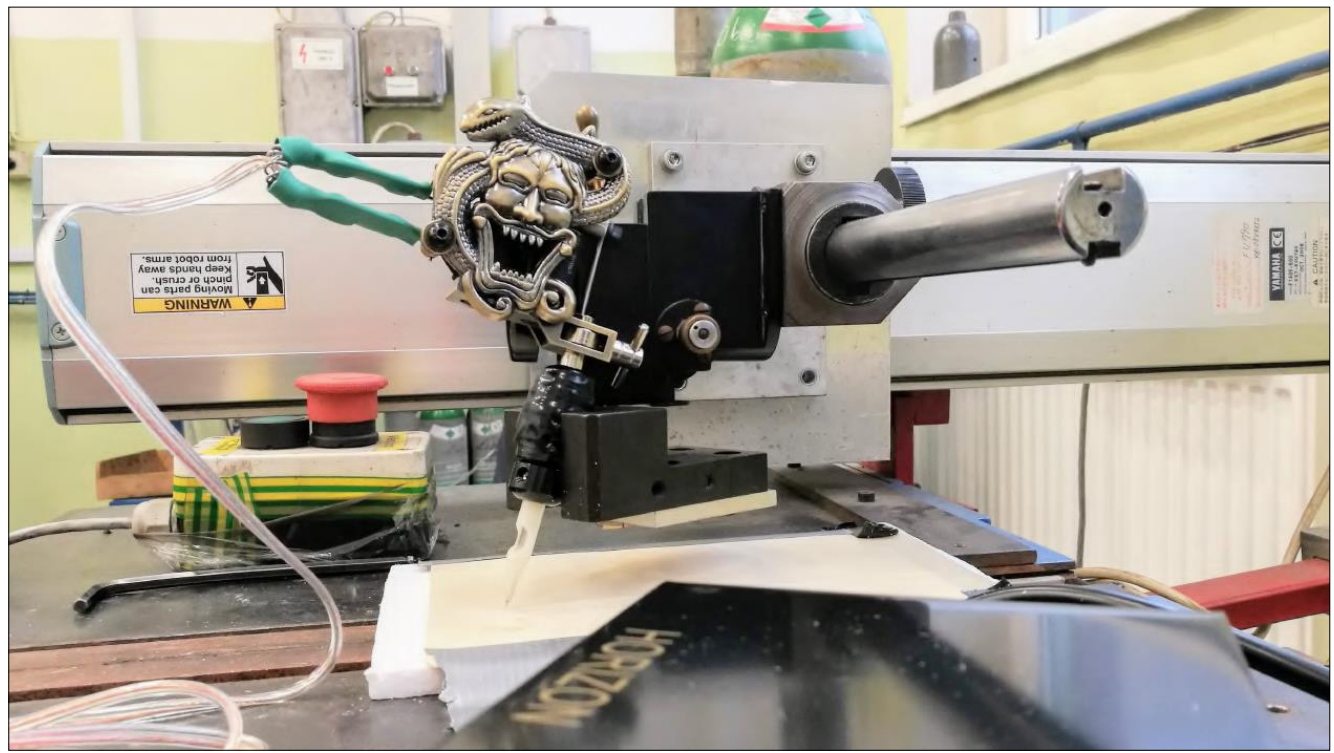

Figure 4. Equipment used for the automation of the tattooing process.

\section{Results}

\subsection{In vivo damaged needles}

Scanning electron microscopy (Zeiss EVO MA10) images provide the most information on needle tip destruction and detachment rates. Strong wear of the needle tips was observed in the samples, the tips of almost all members of the needle group were plastically deformed (Figures 5-7.).

Material composition analysis was also performed by energy dispersive X-ray spectrometry (EDAX Metek Elect Plus). As a result of the study, it was found that the material of the needles is not made of 316L commercially marked austenitic stainless steel, but of a more general, not very biocompatible alloy, 1.4301 steel. The stress of stabbing the skin is abrasive, the wear properties of these steel types are not outstanding [11, 12]. Poor wear resistance in medical devices is unfavourable, as particles detached during the wear process can cause irritation if they enter the body, especially the two main alloys of the steel type: chromium and nickel [13].

\subsection{In vitro experiments}

At lower magnification stereo and electron microscopy images, there is almost no damage to the needle ends. Even at higher magnification, the needles on the training skin showed dulling only on the needle tips used by hand, and with the result shown in Figure 8, no significant damage could be observed on the needles used with the

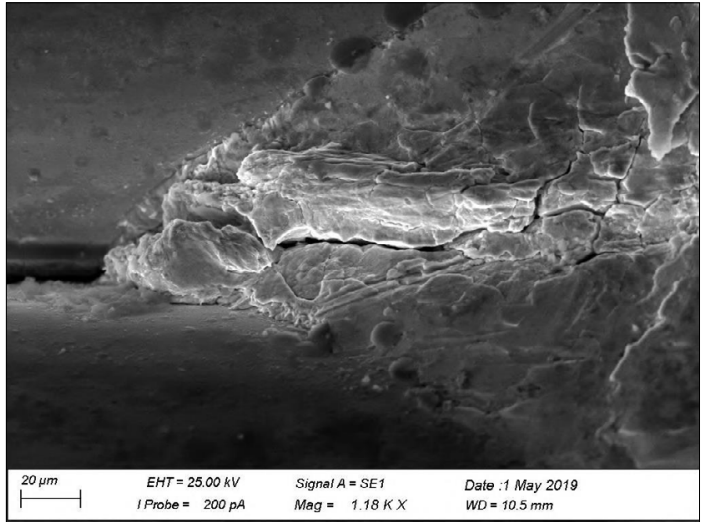

Figure 5. Damage on the soldering of the needle groups in in vivo case.

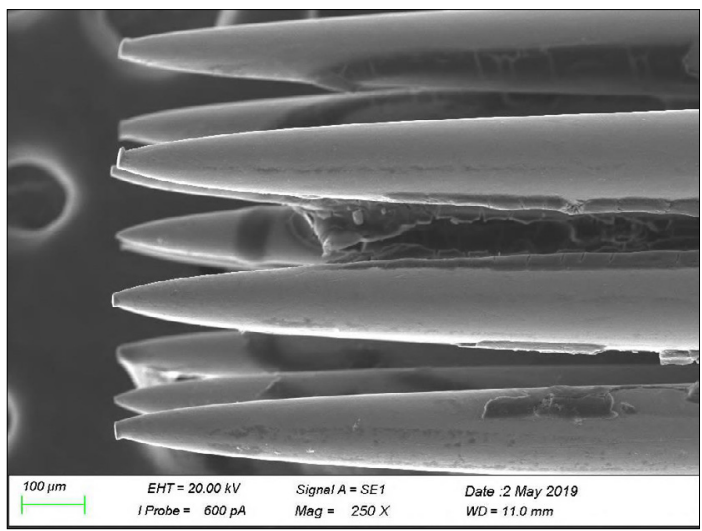

Figure 6. Significant wear was observed in almost all of the needle groups in the in vivo case. 


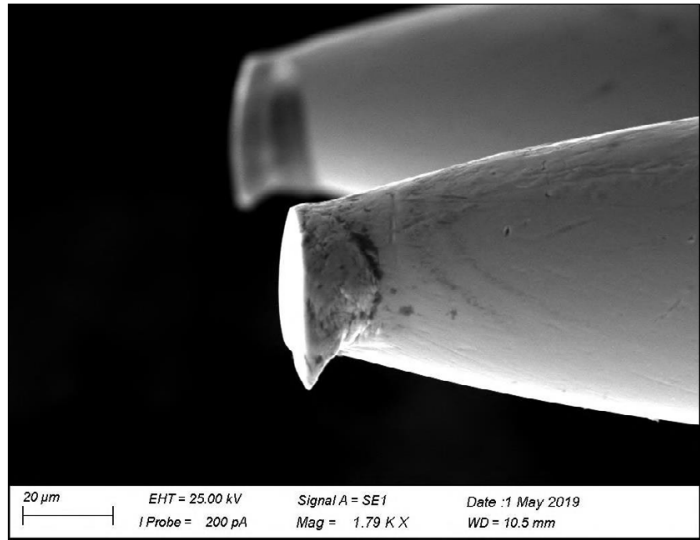

Figure 7. Electron micrograph of a worn needle tip at higher magnification.

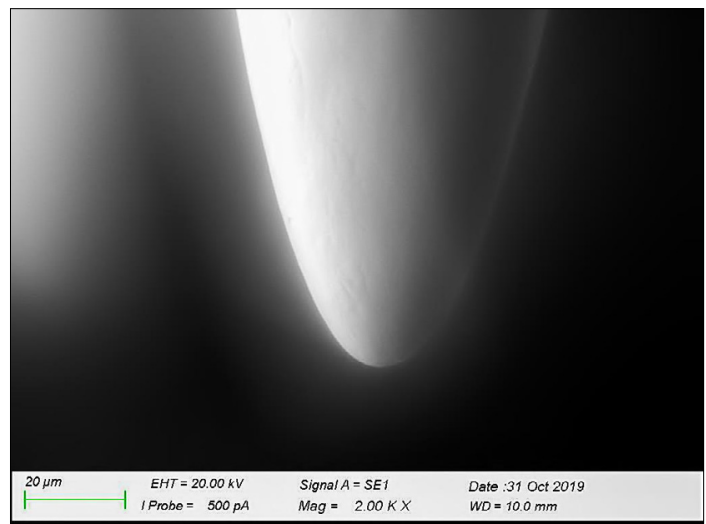

Figure 9. In vitro needle damage in the case of an automated tattoo process on training skin.

linear welding robot, regardless of the duration of the run (Figure 9.). The training skin is made of silicone and is much softer to the touch than real skin, which may have caused the results of real-world tests to differ from those seen in vitro. During the automated tattooing process, however, the soldering of the needles was significantly damaged because the surface of the silicone training skin was easily torn and the needle tips became stuck in it more easily during movement.

\section{Conclusions}

Our studies have confirmed that the tip of tattoo needles as well as the soldering of the needles suffer significant damage even after short-term use. We have found that the properties of the socalled training skin recommended for tattoo artists differ significantly from the properties of real skin, so it is not applicable in laboratory testing

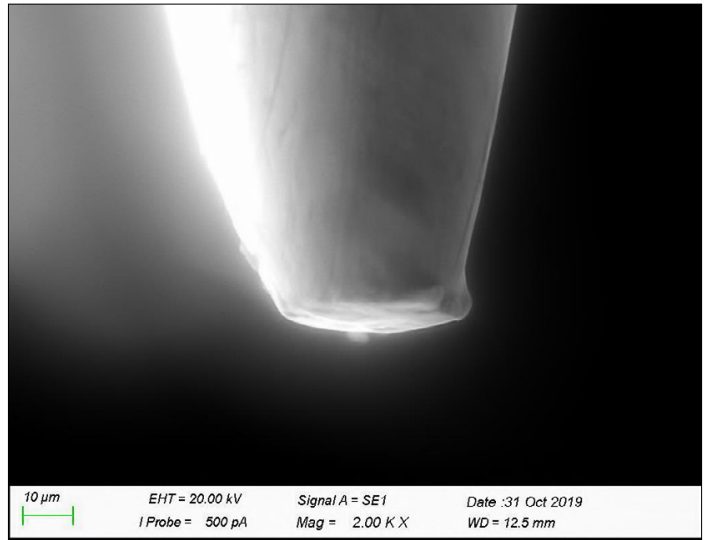

Figure 8. In vitro needle damage in case of handmade process.

for damage to tattoo needles. As a continuation of our research, we would perform the method presented here on pigskin to explore the process of needle wear in more detail as a function of the time elapsed with the tattoo.

\section{Acknowledgments}

The publication was supported by the Ministry of Human Resources' NTP-SZKOLL-19-066 National Talent Program.

\section{References}

[1] Sad B.:. New technologies for dynamic tattoo art. Proceedings of the $5^{\text {th }}$ International Conference on Tangible Embedded and Embodied Interaction. 2011. 313-316.

https://doi.org/10.1145/1935701.1935774

[2] Serup J., Bäumler W.: Tattoo Infections, Personal Resistance, and Contagious Exposure through Tattooing. Current Problems in Dermatology. Basel, Karger 52. (2017) 30-41.

https://doi.org/10.1159/000450777

[3] Sweeney S. M.: Tattoos: a review of tattoo practices and potential treatment options for removal. Current Opinion in Pediatrics, 18/4. (2006) 391-395.

https://doi.org/10.1097/01.mop.0000236388. 64333.cd

[4] Jokinen M. J. et al.: Deformed spinal needle tips and associated dural perforations examined by scanning electron microscopy. Acta Anaesthesiologica Scandinavica, 40/6. (1996) 687-690.

https://doi.org/10.1111/j.1399-6576.1996. tb04511.x

[5] Sitzman B. et al.: The Effects of Needle Type, Gauge and Tip Bend on Spinal Needle Deflection. Anasthesia \& Analgesia, 82/2. (1996) 297-301.

https://doi.org/10.1097/00000539-19960200000014 
[6] Benham M.: Spinal needle damage during routine clinical practice. Anaesthesia, 51. (1996) 843-845. https://doi.org/10.1111/j.1365-2044.1996. tb12614.x

[7] Rout P. G. J. et al.: An Investigation of the Effect on 27-gauge Needle Tips following a Single Local Anaesthetic Injection. Dental Update, 30/7. (2003) 370-374. https://doi.org/10.12968/denu.2003.30.7.370

[8] Kline D., Kuhn T.: Needle Reuse and Tip Damage. Diabetes Care, 27/2. (2004) 617-617. https://doi.org/10.2337/diacare.27.2.617

[9] Abolhassani N., Patel R.: Deflection of a Flexible Needle during Insertion into Soft Tissue. 2006 International Conference of the IEEE Engineering in Medicine and Biology Society. https://doi.org/10.1109/iembs.2006.259519
[10] Kataoka H. et al.: A Model for Relations Between Needle Deflection, Force, and Thickness on Needle Penetration. Lecture Notes in Computer Science, (2001) 966-974. https://doi.org/10.1007/3-540-45468-3_115

[11] Barcelos M. et al.: Wear resistance of AISI 304 stainless steel submitted to low temperature plasma carburizing. REM - International Engineering Journal, 70. (2017) 293-298. https://doi.org/10.1590/0370-44672016700094.

[12] Reza Bateni M. et al.: Wear and corrosion wear of medium carbon steel and 304 stainless steel. Wear, 260/1-2. (2006). 116-122. https://doi.org/10.1016/j.wear.2004.12.037

[13] Schriever I. et al.: Distribution of nickel and chromium containing particles from tattoo needle wear in humans and its possible impact on allergic reactions. Part Fibre Toxicol, 16/33. (2019) https://doi.org/10.1186/s12989-019-0317-1 